\title{
Novel SISO Detection Algorithms for Nonlinear Satellite Channels
}

\author{
Giulio Colavolpe, Senior Member, IEEE, and Amina Piemontese, Member, IEEE
}

\begin{abstract}
We propose novel detection algorithms for linear modulations transmitted over nonlinear satellite channels. These algorithms are derived by using a Volterra-series expansion of the useful signal and by applying the sum-product algorithm to a suitably-designed factor graph. Being soft-input soft-output (SISO) in nature, the proposed detectors can be adopted in turbo processing without additional modifications. When compared with the optimal detection algorithm for these channels, whose complexity is exponential in the channel memory, the proposed schemes result very appealing in terms of tradeoff between performance and computational complexity. Particularly, they can approach the optimal performance with a complexity only linear in the channel memory.
\end{abstract}

Index Terms-Nonlinear satellite channels, Soft-input Softoutput (SISO) detection, turbo detection.

\section{INTRODUCTION}

W E consider a satellite system where nonlinear distortions may originate from the presence of a high power amplifier (HPA) and can cause significant performance impairments. We assume a realistic model and consider a typical satellite channel consistent with the DVB-S2 standard [1].

Many efforts in the literature of the last decades have been devoted to nonlinear channel compensation techniques that can be applied at the transmitter side, such as signal predistortion [2], [3] and data predistortion [4]. In this paper, we consider a different approach to minimize the effect of nonlinearities to the overall system performance, and investigate possible low-complexity detection algorithms to be employed as inner detector in a turbo-equalization scheme. The algorithms must be able to cope with a possibly large intersymbol interference (ISI) introduced by input and output multiplexing (IMUX and OMUX) filters placed before and after the HPA. Assuming that the transfer characteristics of the HPA are perfectly known at the receiver, the optimal maximum-a-posteriori (MAP) symbol detector for this nonlinear channel is perfectly defined. However, it exhibits an exponential complexity in the channel memory and is prohibitively complex for practical purposes. Hence, alternative low-complexity suboptimal schemes require to be investigated. Similarly to low-complexity detection schemes designed in the literature for linear ISI channels [5], detection algorithms for nonlinear channels are based on a Gaussian approximation of the (linear and nonlinear) ISI

Manuscript received September 26, 2011. The associate editor coordinating the review of this letter and approving it for publication was $\mathrm{H}$. Ochiai.

The authors are with Consorzio Nazionale Interuniversitario per le Telecomunicazioni (CNIT) and Università di Parma, Dipartimento di Ingegneria dell'Informazione, Viale G. P. Usberti, 181A, I-43100 Parma, Italy (e-mail: giulio@unipr.it).

Parts of this paper were presented at the IEEE Global Telecommunications Conference, Houston, TX, U.S.A., December 2011. This work is funded by the European Space Agency, ESA-ESTEC, Noordwijk, The Netherlands, under contract no. 4000102300.

Digital Object Identifier 10.1109/WCL.2012.120211.110040 term (e.g., see [6], [7]). Surprisingly, in these works only one sample per symbol interval is employed at the receiver although often it does not represent a sufficient statistic. In [6], the resulting detection algorithm has a complexity which is linear in the channel memory. However, the Gaussian approximation of the (linear and nonlinear) ISI terms with a variance independent of the considered time instant, although varying with the iterations and computed starting from the soft-outputs provided by the decoder, yields a performance which results to be inadequate when the ISI term is significant. On the other hand, the algorithm in [7] relies on a more accurate signal model, based on a Volterra-series expansion [8], but has a much larger complexity (at least quadratic in the channel memory).

We investigate new detectors able to cope with the joint effects of nonlinearities and ISI. They rely on a Volterraseries expansion of the satellite channel, largely adopted in the past for modeling digital satellite channels and for designing equalizers and predistorters [8], [9], and on the use of the framework based on factor graphs (FGs) and the sum-product algorithm (SPA) [10]. The derived algorithms result to be similar, in structure, to those described in [11].

\section{System Model}

Let us consider a linear modulation, with a properly normalized shaping pulse $p(t)$ and symbol interval $T$, and assume that the transmitted symbols $\left\{x_{n}\right\}$, belonging to a given zeromean $M$-th order complex constellation, are independent, uniformly distributed, and normalized to have unit power. The transponder model, shown in Fig. 1, is composed of an IMUX filter $h_{i}(t)$, aimed at removing the adjacent channels, a HPA, and an OMUX filter $h_{o}(t)$ which counters the spectral broadening caused by the nonlinear amplifier. The HPA is a nonlinear memoryless device. The resulting system is nonlinear with memory and describes a "single-carrier per transponder" operation. The received signal is also corrupted by additive white Gaussian noise whose low-pass equivalent $w(t)$ has power spectral density $2 N_{0}$. The low-pass equivalent of the received signal has thus expression

$$
r(t)=s(t)+w(t)
$$

where $s(t)$ is the signal at the output of the OMUX filter.

An approximate model for signal $s(t)$ is based on a Volterraseries expansion [8]. Assuming the satellite channel static and perfectly known at the receiver, we define the impulse response of the system up to the HPA as $h(t)=p(t) \otimes h_{i}(t)$, where symbol $\otimes$ denotes "convolution". Signal $x(t)$ at the HPA input will read

$$
x(t)=\sum_{k} x_{k} h(t-k T)
$$




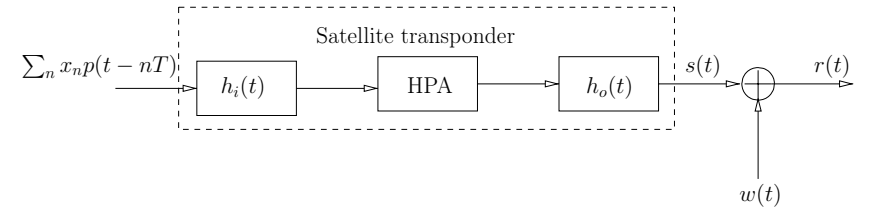

Fig. 1. System model.

whereas signal $y(t)$ at the HPA output can be expressed as a function of $x(t)$ by using the following polynomial expansion in which only odd-order terms appear [8]

$$
y(t)=\left[\sum_{m} \gamma_{2 m+1} \frac{1}{2^{2 m}}\left(\begin{array}{c}
2 m+1 \\
m
\end{array}\right)|x(t)|^{2 m}\right] x(t) .
$$

In (2), parameters $\gamma_{i}$ are complex to take into account for the phase distortions induced by the nonlinear device. With good approximation, the HPA can be modeled as a cubic nonlinearity and we can rewrite (2) as

$$
\begin{aligned}
& y(t) \simeq \gamma_{1} \sum_{k} x_{k} h(t-k T) \\
& +\frac{3}{4} \gamma_{3} \sum_{i} \sum_{j} \sum_{\ell} x_{i} x_{j} x_{\ell}^{*} h(t-i T) h(t-j T) h^{*}(t-\ell T) .
\end{aligned}
$$

In (3), we have used (1) to explicit the dependence on the transmitted symbols and to separate linear and nonlinear effects. The signal $y(t)$ is then filtered through the OMUX filter. Hence, signal $s(t)$ can be expressed as

$$
\begin{aligned}
& s(t) \simeq \gamma_{1} \sum_{k} x_{k} h^{(1)}(t-k T) \\
& +\frac{3}{4} \gamma_{3} \sum_{i} \sum_{j} \sum_{\ell} x_{i} x_{j} x_{\ell}^{*} h^{(3)}(t-i T, t-j T, t-\ell T),
\end{aligned}
$$

where

$$
\begin{gathered}
h^{(1)}(t)=h(t) \otimes h_{o}(t), \\
h^{(3)}\left(t_{1}, t_{2}, t_{3}\right)=\int_{-\infty}^{\infty} h_{o}(\tau) h\left(t_{1}-\tau\right) h\left(t_{2}-\tau\right) h^{*}\left(t_{3}-\tau\right) d \tau .
\end{gathered}
$$

When multiplied by $\gamma_{1}$ and $\frac{3}{4} \gamma_{3}, h^{(1)}(t)$ and $h^{(3)}\left(t_{1}, t_{2}, t_{3}\right)$ take the form of the so-called Volterra kernels of first and third order, respectively. In the following, in order to derive low-complexity detection algorithms, we introduce a further approximation. In the triple summation in (4), we only take the terms for $i=\ell$ or $j=\ell$, and obtain

$s(t) \simeq \sum_{k} x_{k}\left[\gamma_{1} h^{(1)}(t-k T)+\frac{3}{4} \gamma_{3} \sum_{i}\left|x_{i}\right|^{2} \bar{h}^{(3)}(t-i T, t-k T)\right]$,

where

$$
\begin{aligned}
& \bar{h}^{(3)}\left(t_{1}, t_{2}\right)=h^{(3)}\left(t_{2}, t_{1}, t_{1}\right) \\
& +h^{(3)}\left(t_{1}, t_{2}, t_{1}\right)-I\left(t_{1}-t_{2}\right) h^{(3)}\left(t_{2}, t_{2}, t_{2}\right) .
\end{aligned}
$$

In (6), $I(t)$ is an indicator function, equal to one if $t=0$ and to zero otherwise.

The extension to the case of fifth-order nonlinearity model will be discussed later.

\section{Detection Algorithms}

When the system is assumed to have finite memory, the optimal receiver based on the MAP sequence or symbol detection strategy consists of a bank of filters followed by a proper trellis processor. The number of trellis states, and thus the complexity of optimal receiver, is exponential in the channel memory $L$. We point out that, in principle, the real channel memory can be much larger that that assumed by the receiver - the choice of $L$ is often dictated by implementation complexity reasons. In the following, we will consider phase shift keying (PSK) and amplitude/phase shift keying (APSK) modulations typically employed in satellite transmissions [1].

1) PSK modulations: In this case, being $\left|x_{n}\right|^{2}=1$, the signal (5) becomes

$$
s(t) \simeq \sum_{k} x_{k} \bar{h}(t-k T),
$$

where $\bar{h}(t)=\gamma_{1} h^{(1)}(t)+\frac{3}{4} \gamma_{3} \sum_{i} \bar{h}^{(3)}(t-i T, t)$. The channel can thus be approximated as a linear channel with ISI and related detection algorithms employed accordingly. In particular, the receiver front end is represented by a filter matched to pulse $\bar{h}(t)$. When the channel memory $L$ is large, an excellent performance/complexity tradeoff is provided by the suboptimal graph-based algorithm described in [11], which has been derived by applying the SPA to a suitably-designed FG, and is characterized by a complexity that grows only linearly with the channel memory. The algorithm can be applied to the signal model (7) with no modifications.

2) APSK modulations: Digital communication systems typically adopt higher-order amplitude/phase modulations for spectrally efficient applications. However, such modulations have large envelope fluctuations that cause non-negligible distortions when the signal is amplified. To mitigate this detrimental effect, DVB-S2 systems use multilevel APSK constellations which have lower peak-to-average-power ratio and good performance in nonlinear environments. Nevertheless, unlike PSK constellations, APSKs are affected by the so-called constellation centroids warping [12], which is responsible for a reduction in the distance among APSK rings and a differential phase rotation among them. If we still consider the approximate Volterra representation of the useful signal (5) and approximate the terms $\left|x_{i}\right|^{2}$ with $\mathrm{E}\left\{\left|x_{i}\right|^{2}\right\}=1$, this approximation leads to the same low-complexity algorithm described for PSK modulations. Simulation results show that this suboptimal detector exhibits a large performance degradation with respect to the optimal detector. For this reason, we look for more efficient receivers, still designing detection algorithms by using the FG/SPA framework. We introduce a novel FG describing the nonlinear system and apply the SPA to this graph to compute approximate $a$ posteriori probabilities (APPs) of the transmitted symbols. We rewrite the signal (5) using the approximation $\left|x_{i}\right|^{2}=\mathrm{E}\left\{\left|x_{i}\right|^{2}\right\}$ only for $i \neq k$ and preserve the term $\left|x_{k}\right|^{2}$, which helps the detection algorithm to take into account of the warping effect suffered by the constellation points. The new signal model assumed by the receiver becomes

$$
s(t)=\sum_{k} x_{k}\left[g^{(1)}(t-k T)+\left|x_{k}\right|^{2} g^{(3)}(t-k T)\right],
$$


where

$$
\begin{aligned}
& g^{(1)}(t)=\gamma_{1} h^{(1)}(t)+\frac{3}{4} \gamma_{3} \sum_{i \neq 0} \bar{h}^{(3)}(t-i T, t) \\
& g^{(3)}(t)=\frac{3}{4} \gamma_{3} \bar{h}^{(3)}(t, t) .
\end{aligned}
$$

We assume to have a proper set of sufficient statistics, extracted from the received signal, collected in a vector $\mathbf{r}$ of proper length. MAP symbol detection of symbols $\left\{x_{n}\right\}$ requires the evaluation of the APPs $P\left(x_{n} \mid \mathbf{r}\right)$. We can factorize the probability mass function of the transmitted sequence as

$$
P(\mathbf{x})=\prod_{n=0}^{N-1} P_{n}\left(x_{n}\right)
$$

where $P_{n}\left(x_{n}\right)$ is the a priori probability that the symbol $x_{n}$ is transmitted with index $n$. The conditional probability density function of $\mathbf{r}$ given the modulation symbols $\mathbf{x}$ is

$$
p(\mathbf{r} \mid \mathbf{x}) \propto \exp \left(-\frac{1}{2 N_{0}} \int_{-\infty}^{\infty}|r(t)-s(t)|^{2} d t\right) .
$$

We consider a detector designed assuming that the useful signal component $s(t)$ is given by (8). Hence, substituting (8) in (9), defining the following coefficients

$$
\begin{aligned}
h_{i}^{(1)} & =\int_{-\infty}^{\infty} g^{(1)}(t) g^{(1)^{*}}(t-i T) d t \\
h_{i}^{(3)} & =\int_{-\infty}^{\infty} g^{(3)}(t) g^{(3)^{*}}(t-i T) d t \\
h_{i}^{(1,3)} & =\int_{-\infty}^{\infty} g^{(3)}(t) g^{(1)^{*}}(t-i T) d t \\
r_{i}(\beta) & =\int_{-\infty}^{\infty} r(t)\left[g^{(1)^{*}}(t-i T)+\beta g^{(3)^{*}}(t-i T)\right] d t
\end{aligned}
$$

and the following functions

$$
\begin{aligned}
& F_{n}\left(x_{n}\right)=\exp \left[\frac { 1 } { N _ { 0 } } \operatorname { R e } \left\{x_{n}^{*} r_{n}\left(\left|x_{n}\right|^{2}\right)-\frac{\left|x_{n}\right|^{2}}{2} h_{0}^{(1)}\right.\right. \\
& \left.\left.\left.-\frac{\left|x_{n}\right|^{6}}{2} h_{0}^{(3)}-\left|x_{n}\right|^{4} h_{0}^{(1,3)}\right)\right\}\right] \\
& I_{m}\left(x_{n}, x_{n-m}\right)=\exp \left[-\frac{1}{N_{0}} \operatorname{Re}\left\{x _ { n } ^ { * } x _ { n - m } \left(h_{m}^{(1)}\right.\right.\right. \\
& \left.\left.\left.+\left|x_{n}\right|^{2}\left|x_{n-m}\right|^{2} h_{m}^{(3)}\right)\right\}\right] \text {, } \\
& H_{m}\left(x_{n}, x_{n-m}\right)=\exp \left[-\frac{1}{N_{0}} \operatorname{Re}\left\{x_{n}^{*} x_{n-m}\left|x_{n-m}\right|^{2} h_{m}^{(1,3)}\right\}\right]
\end{aligned}
$$

we can factorize the joint APP of the transmitted sequence as

$$
\begin{aligned}
& P(\mathbf{x} \mid \mathbf{r}) \propto P(\mathbf{x}) p(\mathbf{r} \mid \mathbf{x}) \\
\propto & \prod_{n=0}^{N-1}\left[P_{n}\left(x_{n}\right) F_{n}\left(x_{n}\right) \prod_{\ell=1}^{L} I_{\ell}\left(x_{n}, x_{n-\ell}\right) \prod_{\substack{m=-L \\
m \neq 0}}^{L} H_{m}\left(x_{n}, x_{n-m}\right)\right],
\end{aligned}
$$

where $L$ is the assumed channel memory. The corresponding factor graph is depicted in Fig. 2 for the case $L=2$. Nodes $G_{i}$ in the graph collect all factors in (10) which depend on the same two symbols. As an example, we have

$$
G_{2}\left(x_{8}, x_{6}\right)=I_{2}\left(x_{8}, x_{6}\right) H_{2}\left(x_{8}, x_{6}\right) H_{-2}\left(x_{6}, x_{8}\right) .
$$

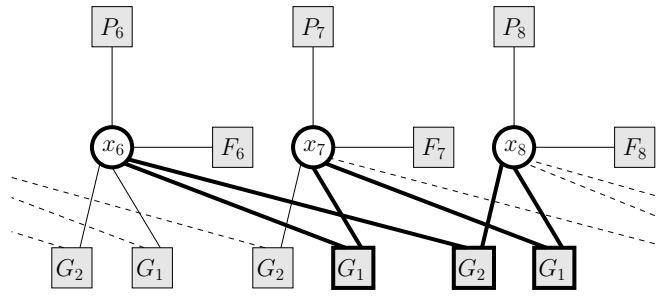

Fig. 2. Three sections of the factor graph corresponding to (10).

This FG has the same structure of the graph considered in [11] for linear channels although the factors are different. It has cycles and the application of the SPA to it leads to an approximate marginalization of (10). However, it is easy to prove that it cannot contain any cycle of length lower than six, irrespectively of $L$. Hence, the SPA can be confidently adopted, since it generally provides a good approximation of the exact marginalizations when the length of the cycles is at least six [10]. The algorithm resulting from the application of the SPA to the described FG has a complexity which is linear in $L$. This is related to the adopted factorization having the appealing property that nodes $G_{i}$, whose number linearly increases with the $L$, have degree two (i.e., they have two edges) independently of channel memory. Due to the presence of cycles in the FG, the SPA cannot lead to a unique schedule nor to a unique stopping criterion for message passing [10]. Among various possible algorithms deriving from different schedules, we adopt a parallel-schedule SPA [11].

Extension to the fifth-order nonlinearity model: We can model the HPA as a nonlinearity of the fifth order and write the signal $s(t)$ assumed by the receiver as

$s(t)=\sum_{k} x_{k}\left[g^{(1)}(t-k T)+\left|x_{k}\right|^{2} g^{(3)}(t-k T)+\left|x_{k}\right|^{4} g^{(5)}(t-k T)\right]$

where

$$
\begin{aligned}
g^{(1)}(t) & =\gamma_{1} h^{(1)}(t)+\frac{3}{4} \gamma_{3} \sum_{i \neq 0} \bar{h}^{(3)}(t-i T, t) \\
& +\frac{5}{8} \gamma_{5} \sum_{n \neq 0} \sum_{m \neq 0} \bar{h}^{(5)}(t-n T, t-m T, t), \\
g^{(3)}(t) & =\frac{3}{4} \gamma_{3} \bar{h}^{(3)}(t, t), \quad g^{(5)}(t)=\frac{5}{8} \gamma_{5} \bar{h}^{(5)}(t, t, t) .
\end{aligned}
$$

The pulse $\bar{h}^{(5)}\left(t_{1}, t_{2}, t_{3}\right)$ is proportional to the approximate fifth-order Volterra kernel. The approximation consists of holding only the terms in the form $\left|x_{p}\right|{ }^{4} x_{q}$ among the 5 -symbols products $x_{i} x_{j}^{*} x_{\ell} x_{n}^{*} x_{m}$ which appear in the polynomial expansion. This new signal model can lead to the same factorization (10) of the joint APP of the transmitted sequence and hence to the same FG of Fig. 2, where factor nodes $G_{i}$ still connect only two symbols. The expressions of the relevant factors in the graph are more involved but the computational load per iteration remains unmodified.

\section{Simulation Results}

We consider coded transmissions with iterative detection/decoding at the receiver side [5], where an iteration consists of a single local iteration of the detector and a single activation of the decoder. We consider channel coding and 


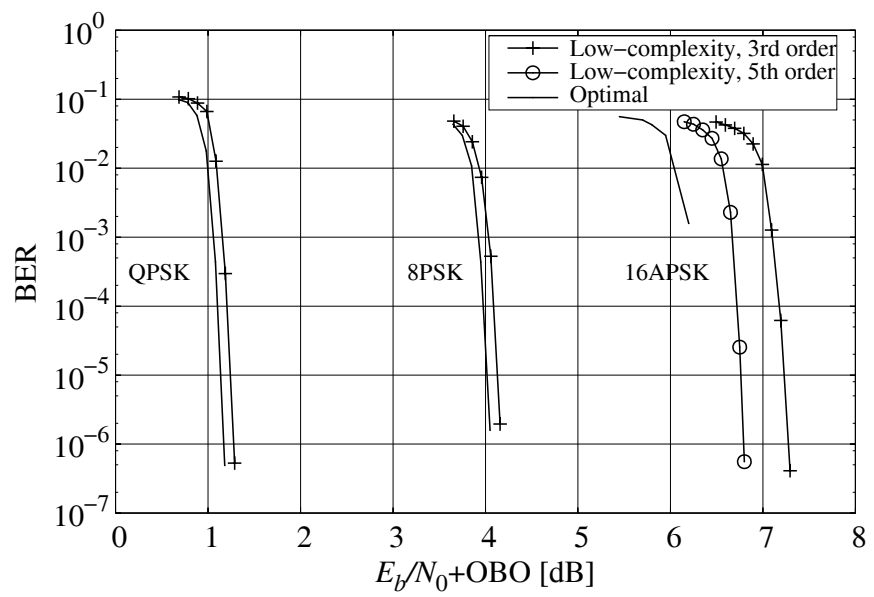

Fig. 3. BER curve for QPSK rate-1/2, 8 PSK rate-2/3 and 16APSK rate$3 / 4$.

modulation formats in the DVB-S2 standard. In particular, we assume that QPSK, 8PSK, and 4+12-APSK modulations are adopted with low-density parity-check codes of rate $1 / 2$, $2 / 3$ and $3 / 4$, respectively. In all cases, we use codewords of length 64800 bits and a maximum of 50 iterations is allowed. At the transmitter, pulse $p(t)$ has a root raised cosine-shaped spectrum with roll-off 0.3 , and the IMUX and OMUX filters and the nonlinear transfer characteristics of the HPA amplifier are given in [1, Figures H.13 and H.12]. In the case of QPSK and $8 \mathrm{PSK}$, the $3 \mathrm{~dB}$-bandwidth of the IMUX/OMUX filters is $1.15 / T$, and it is $1.35 / T$ for the $4+12$-APSK modulation. For each modulation format and for each considered receiver, we optimized the working point of the amplifier, which is generally given in terms of the output back-off (OBO).

In Fig. 3, we report the bit error rate (BER) performance of the proposed low-complexity algorithms versus $E_{b} / N_{0}+\mathrm{OBO}$, $E_{b}$ being the received signal energy per information bit. The performance of the optimal MAP symbol detector is also shown as a reference benchmark. To limit the receiver complexity, the detectors assume that the memory associated with the interference is of three symbols. Larger values of the channel memory make the simulation of the optimal detector practically unfeasible for the highest order modulations we are considering. The BER results show that for PSK schemes the low-complexity detection algorithm has near-optimal performance, being the loss with respect to the optimal detector about $0.1 \mathrm{~dB}$. The performance loss of the suboptimal algorithm is slightly larger in the case of the 4+12APSK modulation and it is $0.5 \mathrm{~dB}$ for the algorithm based on the fifth-order nonlinearity model. The simulation results related to the optimal detector for 4+12-APSK, yet sufficient for estimating the performance loss due to the proposed algorithms, are incomplete, since it is nearly unfeasible to obtain reliable BER curves for detectors working on a 4096state trellis.

Finally, we assess the computational complexity of the considered detection algorithms implemented in the logarithmic domain. We assume that the computation of a non linear function is performed by using a look-up table (LUT). The operations performed at the first iterations only have been
TABLE I

COMPUTATIONAL LOAD PER SYMBOL AND PER ITERATION.

\begin{tabular}{c|cc|cc} 
& \multicolumn{2}{|c|}{ Optimal } & \multicolumn{2}{c}{ Low-complexity } \\
& additions & LUT & additions & LUT \\
\hline Q-PSK & 3633 & 699 & 224 & 0 \\
\hline 8-PSK & 59877 & 11767 & 832 & 0 \\
\hline 16-APSK & 970701 & 192495 & 3200 & 0 \\
\hline
\end{tabular}

neglected. As far as the low-complexity algorithm is concerned, for computing messages between two variable nodes in the FG, we also used the approximation $\log \left(e^{x_{1}}+e^{x_{2}}\right) \approx$ $\max \left(x_{1}, x_{2}\right)$, and hence only a difference is required. The number of additions between two real arguments and accesses to LUT per symbol and per iteration is reported in Table I. Note that, since the choice of the nonlinearity order does not impact the computational load per iteration, the table reports only one entry for the APSK case. This table clearly shows that the proposed algorithms have a complexity much lower than that of optimal detector.

\section{COnClusions}

We have proposed suboptimal low-complexity soft-input soft-output detection algorithms for nonlinear channels. Nonlinear channels and linear modulation formats typical of satellite transmissions have been considered. The proposed algorithms exhibit a complexity which increases only linearly with the channel memory and a very convenient performance/complexity trade-off.

\section{REFERENCES}

[1] ETSI EN 301307 Digital Video Broadcasting (DVB); V1.1.2 (200606), Second generation framing structure, channel coding and modulation systems for Broadcasting, Interactive Services, News Gathering and other Broadband satellite applications, 2006. Available: http://www.etsi.org.

[2] G. Karam and H. Sari, "Analysis of predistortion, equalization, and ISI cancellation techniques in digital radio systems with nonlinear transmit amplifiers," IEEE Trans. Commun., vol. 37, pp. 1245-1253, Dec. 1989.

[3] A. N. D'Andrea, V. Lottici, and R. Reggianini, "RF power amplifier linearization through amplitude and phase predistortion," IEEE Trans. Commun., vol. 44, pp. 1477-1484, Nov. 1996.

[4] A. A. M. Saleh and J. Salz, "Adaptive linearization of power amplifiers in digital radio systems," BSTJ, vol. 62, pp. 1019-1033, Apr. 1983.

[5] M. Tüchler, R. Koetter, and A. C. Singer, "Turbo equalization: principles and new results," IEEE Trans. Commun., vol. 55, pp. 754-767, May 2002.

[6] C. E. Burnet and W. G. Cowley, "Performance analysis of turbo equalization for nonlinear channels," in Proc. 2005 IEEE International Symposium on Information Theory.

[7] D. Ampeliotis, A. A. Rontogiannis, K. Berberidis, M. Papaleo, and G. E. Corazza, "Turbo equalization of non-linear satellite channels using soft interference cancellation," in Proc. 2008 Advanced Satellites Mobile Systems Conf.

[8] S. Benedetto and E. Biglieri, "Nonlinear equalization of digital satellite channels," IEEE J. Sel. Areas Commun., vol. 1, pp. 57-62, Jan. 1983.

[9] E. Biglieri, S. Barberis, and M. Catena, "Analysis and compensation of nonlinearities in digital transmission systems," IEEE J. Sel. Areas Commun., vol. 6, pp. 1706-1717, Jan. 1988.

[10] F. R. Kschischang, B. J. Frey, and H.-A. Loeliger, "Factor graphs and the sum-product algorithm," IEEE Trans. Inf. Theory, vol. 47, pp. 498-519, Feb. 2001.

[11] G. Colavolpe, A. Piemontese, and D. Fertonani, "SISO detection over linear channels with linear complexity in the number of interferers," IEEE J. Sel. Topics Signal Process., vol. 5, pp. 1475-1485, Dec. 2011.

[12] E. Casini, R. De Gaudenzi, and A. Ginesi, "DVB-S2 modem algorithms design and performance over typical satellite channels," Intern. J. Satellite Commun. and Networking, vol. 22, pp. 281-318, May/June 2004. 